\title{
Learning Probabilistic Models for Optimal Visual Servo Control of Dynamic Manipulation
}

\author{
Daniel Nikovski and Illah Nourbakhsh \\ The Robotics Institute, Carnegie Mellon University, Pittsburgh, USA, \{danieln,illah\}@ ri.cmu.edu
}

\begin{abstract}
We present an experiment in sequential visual servo control of a dynamic manipulation task with unknown equations of motion and feedback from an uncalibrated camera. Our algorithm constructs a model of a Markov decision process (MDP) by means of grounding states in observed trajectories, and uses the model to find a control policy based on visual input, which maximizes a prespecified optimal control criterion balancing performance and control effort.
\end{abstract}

\section{Introduction}

Most robots currently in use have limited sensing capabilities and achieve their objectives by following preprogrammed routines or by employing immediate feedback controllers. Building such controllers usually requires detailed kinematic and dynamic modelsS of the robot and the task it is performing, as well as significant design effort. In comparison, humans have remarkable natural abilities to acquire skills in highly complex dynamic tasks involving multi-stage decision making guided by visual input, and perform them with minimal energy expense. Much of robotic research has sought to emulate these abilities and endow robots with visual perception, optimal planning over long horizons, and adaptive acquisition of control policies [2].

Incorporating richer sensing, such as vision, into traditional feedback control schemes, further complicates the design effort, because camera models and state observers have to be built as well. The field of visual servo control has emerged as an alternative, aiming to control the system on the basis of features directly computed from images without state estimation [3]. Learning visual servo control has been explored too, primarily for the case of immediate feedback (fixed set-point controllers).

Optimizing a criterion involving a balance between performance and control cost over an infinite horizon has been studied in the field of optimal control [1]. When the equations of motion of the system are linear and the control criterion is quadratic in both the distance to a goal point and the control cost, linear quadratic regulators have been used with much success. However, nonlinear dynamics and more complicated control criteria usually result in intractable systems of differential equations. Learning the equations of motion further complicates the solution. The field of reinforcement learning is specifically concerned with optimal sequential decision making when the dynamics of the controlled system are unknown [10]. Hard control problems such as balancing an inverted pendulum and the Acrobot task have been studied extensively and also implemented on real setups $[4,9]$. The state of these systems, however, is assumed to be completely known, or (in the case of real robots) precisely measurable from joint encoders.

In the experiment reported in this paper, we have attempted to learn a visual servo controller for a dynamical system with rich dynamics, without prior knowledge of either its kinematics and dynamics, or precise camera calibration and lighting models. The algorithm we present accumulates training data in the form of features extracted from images of sampled trajectories of the system's behavior. Aggregated discrete states of a Markov decision process are grounded in these features, and the transition and cost structure of the MDP is computed based on the state aggregation. The optimal policy for the resulting MDP is found by means of policy iteration and subsequently used for controlling the system. Results for various relative costs of control are reported and the found optimal policies for each relative cost are compared.

\section{Control problem}

The task we are considering is related to the Acrobot and inverted pendulum tasks, as well as to the original centrifugal governor problem of James Watt, which started the theoretical analysis of control systems in the $18^{t h}$ century. A hollow ball of radius $75 \mathrm{~mm}$ is attached to the second link of a 2-degrees-of-freedom (DOF) planar robot manipulator with links of length $l_{1}=265 \mathrm{~mm}$ 
and $l_{2}=115 \mathrm{~mm}$, respectively (Fig. 1 ). The two joints move in approximately parallel planes with link offset of $25 \mathrm{~mm}$ and are actuated by two direct-drive servo motors (attached directly to the links without additional gear boxes other than the ones already embedded within the servos) with torques $44.4 \mathrm{oz} / \mathrm{in}$ and $19 \mathrm{oz} / \mathrm{in}$, and speeds $230 \mathrm{~ms} / 60^{\circ}$ and $90 \mathrm{~ms} / 60^{\circ}$, respectively. The cord connecting the attachment point on the second link and the ball is $215 \mathrm{~mm}$ long and has a rigid hollow tube around it, which essentially turns it into a third link attached to the ball and the second link via universal unactuated joints. Taking into account the rotational symmetry of the ball, the system has 6 DOF, only two of which are actuated. The quiescent state of the ball at settings of zero for both joint angles is shown in Fig. 1.

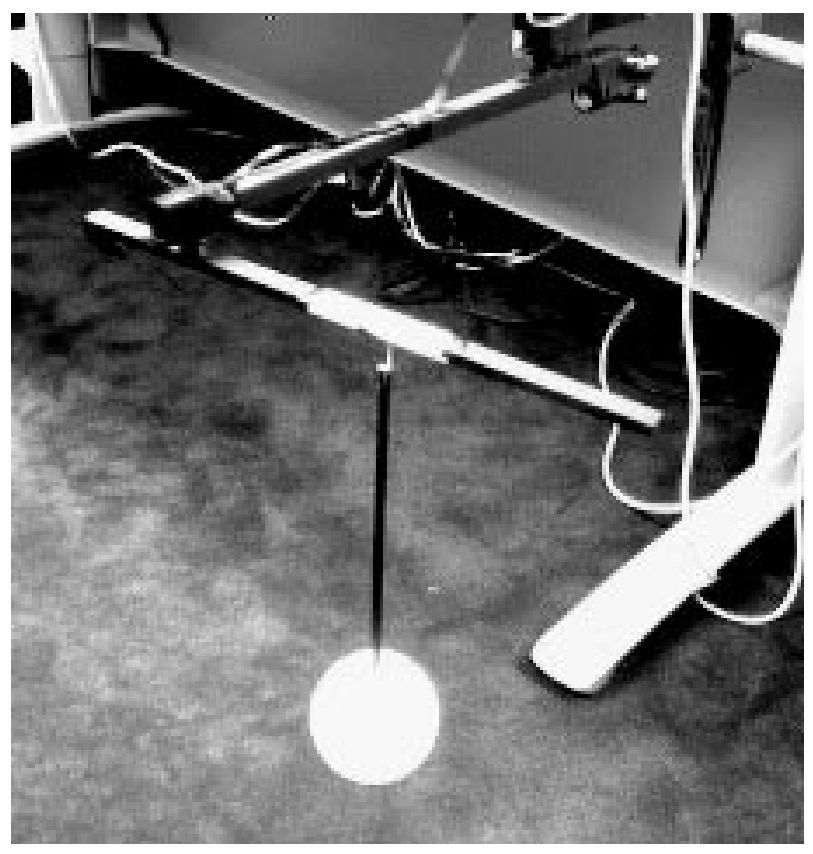

Figure 1: Experimental setup in resting state.

This setup is in fact a spherical pendulum with moving planar support, which has been studied extensively both theoretically and experimentally [6]. Due to the rich dynamics of the system, there is no single control method which can make the pendulum follow arbitrary trajectories. Such a method is not likely to be found, because of the discrepancy between total and actuated degrees of freedom of the system. For this reason, we chose a specific experimental task which effectively limits the degrees of freedom of the system.

The objective of the controller is to move the manipulator so as to keep the ball as high as possible with minimal control effort. The effort is measured by the amount of movement of the joints in servo-motor notches per con- trol step, which in its turn is proportional to the current and power consumed by the motors.

The performance of the controller is inferred from visual input. The manipulator and the ball are observed from a camera placed $1380 \mathrm{~mm}$ above the plane of the motion of the attachment point of the cord to the second link, and pointed approximately perpendicular to that plane. An image is acquired, processed, and a new control position for the servos is determined every $90 \mathrm{~ms}$. The ball is painted in bright yellow color, and a patch of bright pink color is placed at the second link, centered at the attachment point of the ball cord. By tuning the color gains, exposure time, light sensitivity, brightness, and contrast of the camera, it is possible to separate the ball and the patch from the background by thresholding the resulting pixel intensities without any modifications to the regular office lighting conditions. Separating the ball pixels from the patch pixels is based on color information - the red component is larger than the green component for the patch pixels, and vice versa for the ball pixels.

It is the Euclidean distance between the centroids of the two patches in a particular image frame that we take as a measure of how high the ball is with respect to its resting state. The exact height is not measurable directly, but is proportional (non-linearly) to the projection of the third link on the image plane, so the latter is a reasonable substitute measure.

If the measured height in an image frame $t$ is $H_{t}$, and the sum of the displacement of the servo motors is $C_{t}$, the total performance for that frame is $R_{t}=H_{t}-\alpha C_{t}$, where $\alpha \geq 0$ is a user-specified multiplier expressing the relative cost of control. The control objective then is to find a control policy $\pi^{*}$ that maximizes the average performance of the system over an infinite number of control points:

$$
\pi^{*}=\operatorname{argmax}_{\pi} \lim _{T \rightarrow \infty} \frac{1}{T} \sum_{t=1}^{T} H_{t}-\alpha C_{t}
$$

\section{Controller design}

Designing a general-purpose controller for this system, which can bring the ball to any state in its state space is an extremely complicated problem: too few of the joints are actuated, the links are not completely rigid, and the significant mass of the ball in comparison with the servo torques interacts with the links in ways which are hard to capture in a dynamical model. In addition, even though the kinematics of the arm are known, the camera is not calibrated and the actual state of the system cannot be inferred; such a model is not even possible, because estimating the state of a ball flying in three-dimensional space from a single camera image is an ill-posed prob- 
lem. Even if estimating the position was possible, the frame rate $(11 \mathrm{~Hz})$ is too low to estimate the translational and rotational velocities of the ball by frame differencing.

Instead of building a general-purpose controller, our strategy is to force the ball into self-stabilizing trajectories and choose the one which is optimal with respect to both height and control effort. Forcing the ball to circle steadily is a suitable choice of a set of trajectories: we found that for a certain class of open-loop control schedules, the movement of the ball is self-stabilizing around a central orbit and not too sensitive to small control delays. Such trajectories also keep the projection of the ball quite far from the attachment point on the second link, which results in good performance.

One open-loop control which always brings up the ball and holds it in a steady circle is an ellipsoidal motion in joint space with amplitude of the first joint at least 20 notches $\left(14^{\circ}\right)$ and amplitude of the second joint at least 30 notches $\left(21^{\circ}\right)$ and period less than $800 \mathrm{~ms}$. The resulting shape of motion of the attachment point on the second link in Cartesian space is quite far from circular and is even further distorted by the significant centrifugal forces exerted on the ball and transferred to the link via the connecting cord; nevertheless, the resulting motion of the ball is roughly circular. The problem with this control is that it is expensive in terms of control effort and a lot of energy is expended even when the ball is in high orbit and has enough momentum to go on circling with little or no effort. However, starting with controls with lower amplitude never succeeds in bringing the ball into a steady orbit and instead results in chaotic jerky movements close to the starting state. The problem then becomes how to switch efficiently between a powerful, but expensive control, which almost always succeeds in bringing the ball to a high orbit, and a weak control, which is inexpensive, but affects the height of the ball very differently in different situations.

The two controls we considered were $a_{1}$, the ellipsoidal motion with amplitudes 20 and 30 notches for the two joints, which completes a revolution on the ellipse in $720 \mathrm{~ms}$ ( 8 control frames), and $a_{0}$, the motion with zero amplitude, when the joints of the arm remain at their previous positions. Switching between controls is done only after completion of a full revolution, i.e., after 8 control points with the same amplitude. When the ball is in a stable orbit, this switch occurs at approximately the same phase of the orbit.

This is a sequential decision problem, because several actions are required to move the system between different orbits: even when applying $a_{1}$ only, it takes at least 4 revolutions (32 control points, or 2.88 seconds) to enter a stable high orbit from the quiescent state of the ball.
Conversely, if the ball is in a stable orbit, it takes at least 8 revolutions of no action $\left(a_{0}\right)$ for it to drop down close to its resting state. Furthermore, when the ball has moved down past a certain height of the orbit, subsequent applications of $a_{1}$ appear to produce initially only erratic movements, and it takes relatively longer time to bring it back up, in comparison to the case when the ball is starting from the quiescent state. Consequently, the task of the controller is to bring the ball up initially by applying $a_{1}$ several times, and once it is high enough, use the momentum of the ball to conserve energy by applying action $a_{0}$. However, as soon as the ball drops down to a level, which is likely to result in chaotic behavior, the controller should bring it back up by applying action $a_{1}$.

The decision of when to switch the actions could potentially be based on many characteristics of the ball's trajectory, measured from the 8 images taken during one revolution. One possibility is to use the 8 ball locations directly; another one is to use derived statistics such as the average radius of the trajectory, or some estimate of its phase. Clearly, the ball's trajectory during the next revolution depends on all of these variables, but if a compact decision rule is to be found, it is essential to find one or more features to base the decision upon.

Since the performance criterion to be optimized includes the estimated distance $H$ between the center of the ball and the attachment point on the manipulator, we chose to use the average value $\bar{H}$ of $H$ over the last 8 frames as a description of the state of the ball. Clearly, this introduces some perceptual aliasing, because important parameters of the ball's flight are ignored, such as the phase of the trajectory, as well as the location of the individual points along it.

Failure to consider informative aspects of the true state results in decreased determinism of the observed evolution of the state, and higher uncertainty about the effect of actions. In order to deal with this problem, we rely on an algorithm for stochastic optimal control which samples the system by a consistent exploration policy, builds a stochastic MDP model of the state evolution and state rewards, and then uses policy iteration to find the policy that maximizes the average expected reward.

Depending on the kind of visual features observed by the controller, the modeled dynamical system can either be fully or partially observable. Estimating the positions as well as the velocities of the ball and all parts of the manipulator would result in a fully observable system - however, as noted, it is not possible to observe velocities in our experimental set-up. Still, if a long enough sequence of features is used as the state of the system, the resulting process can still be fully observable and the use of a fully-observable MDP is justified. Further discussion on when this is possible is given in [7]. 
During exploration, the system attempts to cover the whole state space of the problem (in this case, all possible heights of orbit), and try all actions everywhere. The result of exploration is a database of transitions of the form $\left(\bar{H}_{i}, a_{i}\right), i=1, N_{o}$, which are assumed to be representative of the dependency of $\bar{H}_{i}$ on $\bar{H}_{i-1}$ and $a_{i}$. The performance $R_{i}$ at time $i$ does not have to be stored explicitly, because it can always be computed as $R_{i}=\bar{H}_{i}-\alpha C\left(a_{i}\right)$.

In order to find an optimal policy, we construct an MDP from the database of transitions. An MDP is described by the tuple $(S, D, A, P, R)$, where $S$ is a set of states of size $N_{s}, D$ is an initial probability distribution over these states, $A$ is a set of actions of size $N_{a}, P$ is a transition function that maps $S \times A$ into probability distributions over $S$, and $R$ is a reward function that maps $S \times A$ into a scalar. The expected average performance of following a particular policy $\pi(s)$, when starting in state $s \in S$ can be computed by solving the linear system of equations for all states

$$
V(s)=R[s, \pi(s)]-\hat{R}+\sum_{s^{\prime} \in S} \operatorname{Pr}\left[s^{\prime} \mid s, \pi(s)\right] V\left(s^{\prime}\right),
$$

where $s^{\prime}$ ranges over the whole state space, and the scalar $\hat{R}$ is the expected average reward of the policy $\pi$ [8]. If the number of states in $S$ is finite, the value function for each state can be uniquely determined - even though there is an extra variable $\hat{R}$ to be found, the value function of the states in $S$ is unique up to an additive constant, so by setting $V\left(s_{0}\right)$ to 0 for some arbitrary state $s_{0}$, we can obtain a completely determined linear system of equations [8].

The policy iteration algorithm proceeds by choosing randomly an initial policy $\pi$, estimating its value function as described above, and then improving the policy by choosing for each state $s$ the action which maximizes its expected performance if started at this state:

$$
\pi^{\prime}(s)=\operatorname{argmax}_{a}\left[R(s, a)+\sum_{s^{\prime} \in S} \operatorname{Pr}\left(s^{\prime} \mid s, a\right) V\left(s^{\prime}\right)\right] .
$$

If $\pi^{\prime}(s)=\pi(s)$, the algorithm terminates, and the optimal policy $\pi^{*}(s)=\pi(s)$; otherwise $\pi(s)$ is set to $\pi^{\prime}(s)$, and the algorithm proceeds with another sequence of policy estimation and improvement steps, until termination.

In order to apply this algorithm to the current control problem, however, we need a discrete state space with relatively few states, and good estimates of the transition function of the MDP under all actions. All we have so far is a database of transitions in continuous feature space, as represented by the feature $\bar{H}_{i}$, which are hopefully representative of the behavior of the system in different modes under different actions.

Our approach is to ground a small set of discrete states into feature space by means of clustering the starting states in the transition database, and subsequently estimating the transition probabilities between clusters under all actions. The rationale behind this approach is that a clustering algorithm naturally produces clusters of states aggregated in regions with high occupancy, which results in approximately even distribution among clusters of the samples, from which transition matrices are estimated, and thus in higher precision of the estimates. On the contrary, a uniform discretization of feature space, which is not driven by the actual experienced trajectories, is likely to result in imprecise frequency counts and thus in high variance of the estimates.

Currently, the algorithm uses a fixed predetermined number $N_{s}$ of clusters. This number depends on the number $N_{o}$ of sampled transitions in the database - if we assume a relatively even distribution of starting states among clusters, then an average of $N_{o} / N_{s}$ states are likely to appear to start from each cluster. Since there are a total of $N_{s}$ clusters, where these transitions can lead to, and $N_{a}$ actions to try, we can expect that each entry in the transition probability tables would be estimated from an average of $N_{o} /\left(N_{a} N_{s}^{2}\right)$ samples. The larger this number, the less variance will exist in these estimates.

The aggregated performance for each aggregated state (cluster) is taken to be the average of the performance for those transitions in the database, whose starting states belong to this cluster. The initial distribution in this case is deterministic - the system always starts in the cluster, to which the quiescent state of the ball is assigned.

\section{Experimental results}

We experimented with different exploration policies, trying to find one which could sample fairly evenly all modes of the system. Randomly choosing actions $a_{0}$ and $a_{1}$ with equal probabilities is not such a policy - under it, the ball stays mostly in two states: either in high steady orbit, or near the resting state, moving chaotically. The system spends the least time in the most interesting part of state space - the boundary between steady high and steady medium-high orbits, where it is very likely to dwell under optimal energy-conserving policies.

To alleviate this bias of completely random exploration towards unimportant parts of state space, we devised an exploration policy which persistently probed the interesting regions of state space. The policy was of the form $a_{1}^{4} a_{0}^{k} a_{1}^{k} a_{0}^{10}, k=1,10$, run over six runs to produce a total of 504 state transitions. Its purpose was to bring the ball up to a steady orbit, and then test for how many revolutions it was safe to let the ball move on momentum only, so that the same number of revolutions with $a_{1}$ could bring it back up, instead of entering a chaotic low orbit, from which it could not recover without expending 
too much effort. The last 10 revolutions of $a_{0}$ ensured that the ball came to a rest before trying the next deeper probing sequence. The resulting transitions are shown in Fig. 2. It can be seen that while action $a_{0}$ fairly consistently reduces the orbit, the consequences of action $a_{1}$ have much more uncertainty, although in general it increases the height of the orbit until that height reaches a limit.

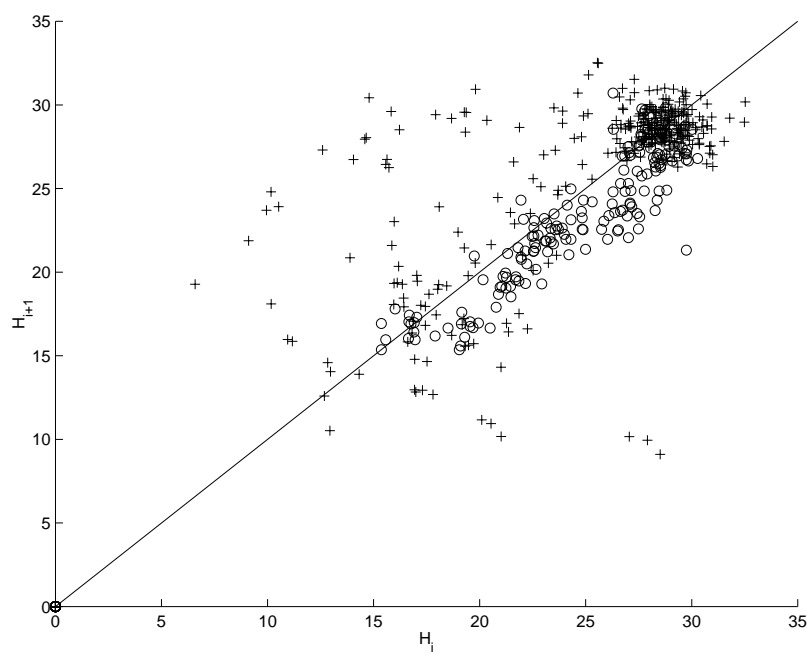

Figure 2: Observed transitions for the exploration policy. Action $a_{0}$ is denoted by 'o', and action $a_{1}$ is denoted by '+'. Transitions below the solid line reduce the height, while those above the line increase it.

We used the transitions experienced during exploration to cluster the starting heights into $N_{s}$ different discrete states by means of k-means clustering, also known as learning vector quantization (LVQ) [5]. The resulting cluster centers (LVQ code book) were used to label both the starting and successor states of each transition (each successor state is the starting state of the next transition in the database). The transition matrices of the MDP were computed by frequency counting: the probability of moving from state $s_{i}$ to state $s_{j}$ under action $a$ was computed as the number of transitions starting in $s_{i}$ and ending in $s_{j}$ under action $a$, divided by the total number of transitions out of $s_{i}$ under $a$. The aggregated performance for state $s$ under action $a$ was the average height during all transitions starting in $s$ under action $a$, less the cost of action $a$ multiplied by the factor $\alpha$, with $C\left(a_{0}\right)=0$ and $C\left(a_{1}\right)=5$.

The MDP was used to compute the optimal action for each of the 10 states by means of policy iteration. Not surprisingly, the optimal policy was threshold-based: for all orbits lower than a threshold $\theta$, the optimal action was $a_{1}$, while for orbits higher than the threshold, the opti- mal action was $a_{0}$. The threshold $\theta$ lies halfway between the centers of the two clusters, where the optimal action changes, and in order to minimize the effect of the random cluster initialization of the LVQ algorithm, five runs were performed and the resulting thresholds averaged. (Note that the number of clusters on the two sides of the threshold is in general different between runs.) The computed optimal policies for various values of $\alpha$ were used to control the arm. The results are shown in Table 1.

Table 1: Results for three values of the cost multiplier $\alpha$. For each value, the average height $\bar{H}$ and cost $\bar{C}$ measured over 1000 revolutions are shown, as well as the predicted total performance $\hat{R}$ as estimated by the policy iteration algorithm, and the actual total performance $\bar{R}=\bar{H}-\alpha \bar{C}$.

\begin{tabular}{|r|r|r|r|r|r|}
\hline$\alpha$ & $\theta$ & $\bar{H}$ & $\bar{C}$ & $\bar{R}$ & $\hat{R}$ \\
\hline 0 & $\infty$ & 28.36 & 5.00 & 28.36 & 28.43 \\
\hline 1 & 27.86 & 26.54 & 3.05 & 23.49 & 24.77 \\
\hline 2 & 27.18 & 25.13 & 2.28 & 20.09 & 21.44 \\
\hline
\end{tabular}

The policy iteration correctly discovers that when there is no cost of control $(\alpha=0)$, the optimal policy is to apply always action $a_{2}$. When $\alpha>0$, the optimal policy maintains a lower orbit, trying to economize effort according to its relative cost. Also, there is fairly good agreement between the predicted performance by the policy iteration algorithm $(\hat{R})$ and the actual performance measured from the test run $(\bar{R})$, which indicates that the stochastic model obtained by grounding discrete states into features is reasonably good. Another look at these data is shown in Fig. 3, where the total performance for all policies is plotted against the relative cost of control. It can be seen that each policy is optimal only around the value of $\alpha$ that it was computed for, and the learning algorithm takes into consideration that cost when computing a control policy. This, of course, does not necessarily mean that these are the exactly optimal policies and better ones cannot be found by some other algorithm.

Fig. 4 shows a sample path of the height of the controlled system under the computed optimal policy for $\alpha=1$, along with the control signal. It can be seen that the controller does not follow an open loop policy, but performs feedback control based on the measured height of the ball from visual input.

\section{Conclusions and future work}

The proposed algorithm for state grounding in image features was able to find a suitable real-time control policy based on visual input for a dynamic manipulation task 


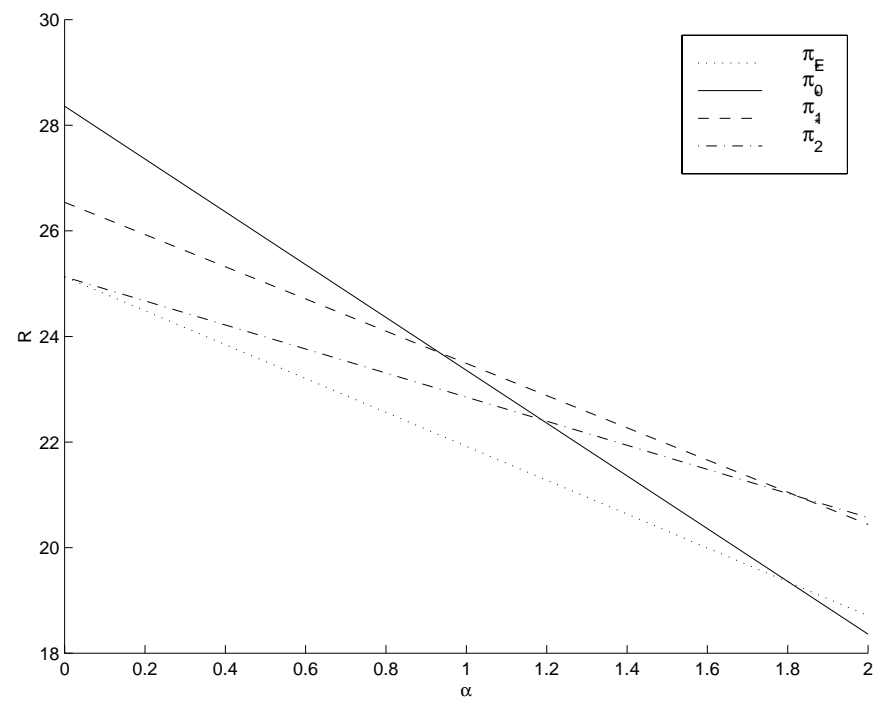

Figure 3: Performance under varying cost multipliers for the exploration policy $\left(\pi_{E}\right)$ and the computed optimal policies $\pi_{0}^{*}, \pi_{1}^{*}, \pi_{2}^{*}$, for $\alpha=\{0,1,2\}$, respectively.
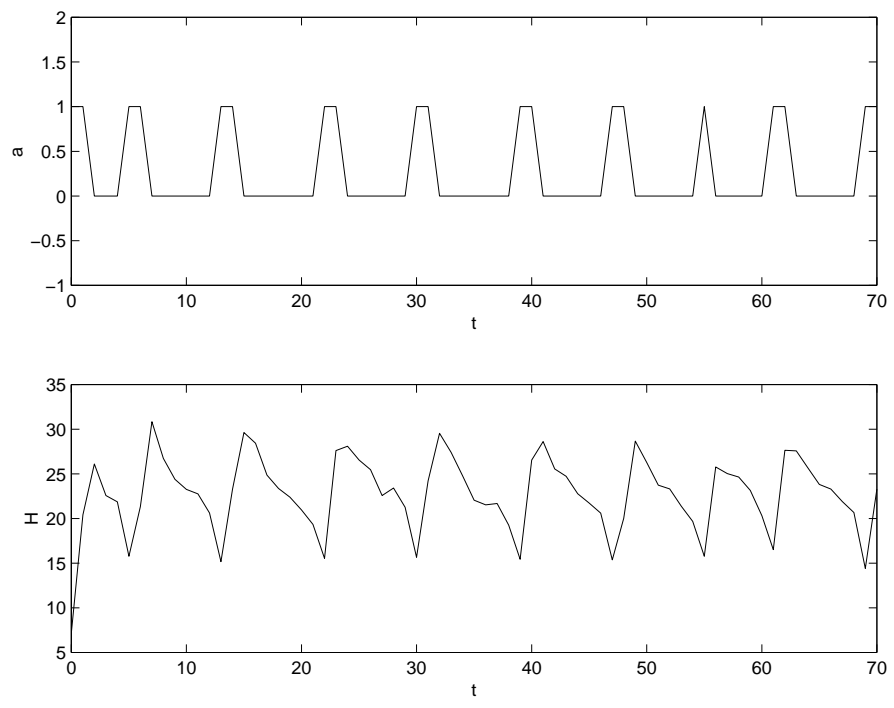

Figure 4: Control signal and measured height of the ball under the computed optimal policy for $\alpha=1$. performed by an underactuated manipulator. The algorithm takes into consideration the relative cost of control to adjust the control policy by varying the threshold $\theta$ of its control rule. The adaptive nature of the state grounding process is essential for the exact determination of this threshold, which varies very little between different cost multipliers and a fixed apriori discretization of the state variable cannot capture these fine differences.

It is likely, however, that including more features representative of the state of the system might produce even better policies. Currently, the controller does not take into consideration the phase of the ball along its orbit this phase visibly varies due to random fluctuations and the interaction of the movement of the manipulator with the natural period of the pendulum the ball is attached to. Furthermore, the controller currently fails to recognize whether the ball is still moving in a steady orbit, or has entered chaotic movement, and it can be expected that the optimal actions in each of these cases would be different. Supplying the standard deviation of the radius of the trajectory $H$ to the learning algorithm might result in better state grounding and hence in better policies.

\section{References}

[1] Dimitri P. Bertsekas. Dynamic Programming and Optimal Control. Athena Scientific, Belmont, Massachusetts, 1995.

[2] J. Connel and S. Mahadevan. Robot learning, 1993.

[3] S. Hutchinson, G. Hager, and P. Corke. A tutorial on visual servo control. IEEE Trans. Robot. Automat., 12(5):651670, 1996

[4] T. T. Jervis and F. Fallside. Pole balancing on a real rig using a reinforcement learning controller. Technical Report CUED/F-INFENG/TR. 115, Cambridge University, UK, 1992.

[5] T. Kohonen. Learning vector quantization. In The Handbook of Brain Theory and Neural Networks, pages 537540. The MIT Press, Cambridge, Massachusetts, 1995.

[6] J. Miles. Damped spherical pendulum. Journal of Sound and Vibrations, 140:327-330, 1993.

[7] D. Nikovski. State-Aggregation Algorithms for Learning Probabilistic Models for Robot Control. PhD thesis, Carnegie Mellon University, The Robotics Institute, February 2002.

[8] Martin L. Puterman. Markov Decision Processes-Discrete Stochastic Dynamic Programming. John Wiley \& Sons, Inc., New York, NY, 1994.

[9] M. Spong. The swingup control problem for the acrobot. IEEE Control Systems Magazine, Feb, Feb. 1995.

[10] R. S. Sutton and A. G. Barto. Reinforcement learning. The MIT Press, Cambridge, Massachusetts, 1998. 